\title{
Multigate Transcranial Doppler Ultrasound System with Real-Time Embolic Signal Identification and Archival
}

\author{
Lingke Fan, Member, IEEE, Enrico Boni, Piero Tortoli, Senior Member, IEEE, \\ and David H. Evans, Senior Member, IEEE
}

\begin{abstract}
An integrated system for acquisition and processing of intracranial and extracranial Doppler signals and automatic embolic signal detection has been developed. The hardware basis of the system is a purpose-built acquisition/processing board that includes a multigate Doppler unit controlled through a computer. The signal-processing engine of the system contains a fast Fourier transform (FFT)-based, spectral-analysis unit and an embolic signaldetection unit using expert system reasoning theory. The system is designed so that up to four receive gates from a single transducer can be used to provide useful reasoning information to the embolic signal-detection unit. Alternatively, two transducers can be used simultaneously, either for bilateral transcranial Doppler (TCD) investigations or for simultaneous intra- and extracranial investigation of different arteries. The structure of the software will allow the future implementation of embolus detection algorithms that use the information from all four channels when a single transducer is used, or of independent embolus detection in two sets of two channels when two transducers are used. The user-friendly system has been tested in-vitro, and it has demonstrated a $\mathbf{9 3 . 6 \%}$ sensitivity for micro-embolic signal (MES) identification. Preliminary in-vivo results also are encouraging.
\end{abstract}

\section{INTRODUCTION}

$\mathrm{D}$ OPPLER ultrasound is a valuable technique for the detection of circulating emboli, whether they are solid or gaseous in nature [1]-[4]. The basis of the detection technique is that, if the acoustic properties of the embolus are sufficiently different from those of the surrounding blood, then when it enters the Doppler sample volume there will be a transient increase in the power of the Doppler signal at a frequency corresponding to the velocity of the embolus through the sample volume [5]. This is not, of course, the only mechanism that can cause a transient rise in Doppler signal power; therefore, careful analysis of the signal is necessary to distinguish between signals arising from embolic events and those arising from other mechanisms such as probe movement or electrical interference. Traditionally, trained human observers have carried out this task, but

Manuscript received July 7, 2005; accepted November 26, 2005. The authors gratefully acknowledge the financial support of the European Union (UMEDS-QLG1-CT-2002-01518).

L. Fan and D. H. Evans are with the Medical Physics Group, Department of Cardiovascular Sciences, University of Leicester, Leicester, UK (e-mail: lingke.fan@le.ac.uk).

E. Boni and P. Tortoli are with the Department of Electronics and Telecommunications, University of Florence, Florence, Italy.

Digital Object Identifier 10.1109/TUFFC.2006.117 there are many difficulties with this approach. Periods of monitoring may extend for several hours, and not only does this put great demands on the concentration of the observer, but it is costly. The environment in which the monitoring must take place is often both busy and noisy, which diminishes the ability of the observer to detect what may be subtle increases in power. And there is considerable evidence that, for many embolic signals, observer reproducibility is poor. Because of these difficulties, various systems have been developed in order to automate the detection task [6]-[18]. But to date no system has been acknowledged as being sufficiently reliable to replace the human observer in the clinical environment. This paper describes the design and implementation of a new automated embolic signal identification and archival system (ESIAS) with flexible features that is intended to overcome some of the drawbacks of currently available systems.

The new system consists of a custom-built board containing both a programmable dual-channel/multigate transcranial Doppler (TCD) unit and a digital signal processor (DSP), performing embolus detection. The embolus detection function was realized using expert system reasoning theory to process information extracted from the time, frequency, energy, and neighborhood domains. The expert system structure was described previously in detail for an off-line implementation [12] and for a unidirectional, real-time implementation using an external TCD machine with a single gate [17]. The latest implementation, with a programmable dual-channel TCD unit, allows the development of algorithms incorporating multigate information from bi-directional Doppler signals. The expectation is that this will further improve the sensitivity and specificity of the system.

A user-friendly, Windows-based system control enables the operator to view, in real time, four spectrograms related to echo-signals from either one or two probes connected to the unit. When an embolic event is detected, the data archival structure of the system automatically stores a pair of one-second long raw audio Doppler signals (forward and reverse flow directions) for each gate. The same structure also allows the manually triggered storage of the raw signals corresponding to the currently displayed sonograms (a pair of 3.6-second segments of raw signal components for each gate), if the operator decides to further investigate what has been displayed on the screen. The system specification is summarized in Table I. 
TABLE I

The Specifications of the ESIAS.

\begin{tabular}{|c|c|}
\hline System & Specification \\
\hline $\begin{array}{l}\text { Ultrasound transmission and reception } \\
\text { (TX-RX) functions }\end{array}$ & $\begin{array}{l}\text { Built-in programmable TCD unit supporting two transmit } \\
\text { channels and four gates }\end{array}$ \\
\hline Microembolic signal identification & $\begin{array}{l}\text { Automated embolic event detection with a detection reso- } \\
\text { lution of } 2.56 \mathrm{~ms}\end{array}$ \\
\hline Signal source for embolic event identification & $\begin{array}{l}\text { The demodulated digital signals from the on-board TCD } \\
\text { unit or the analogue directional signals from an external } \\
\text { Doppler source }\end{array}$ \\
\hline Frequency resolution & Better than $100 \mathrm{~Hz}$ \\
\hline Sonogram display & $\begin{array}{l}\text { Real-time sonograms updated every } 5.12 \mathrm{~ms} \text { for each of } \\
\text { four gates, showing Doppler frequencies for forward and } \\
\text { reverse flow components }\end{array}$ \\
\hline Microembolic event data file & $\begin{array}{l}\text { Eight raw data segments (each of one second duration) } \\
\text { saved for each detected event }\end{array}$ \\
\hline Identification histogram display & $\begin{array}{l}\text { Most recent 4-hour histogram of detected results displayed, } \\
\text { updated every } 10 \text { minutes }\end{array}$ \\
\hline Identification histogram data file & $\begin{array}{l}\text { The number of detected events in every } 10 \text {-minute detec- } \\
\text { tion period for the whole monitoring history saved in a data } \\
\text { file }\end{array}$ \\
\hline System control & User-friendly Windows ${ }^{T M}$ based system control \\
\hline
\end{tabular}

Details of the system design and implementation are given in the next section, which also describes the criteria used for micro-embolic signal (MES) identification and archival. Section III reports on in-vitro tests based on a flow phantom for which both solid and gaseous emboli can be introduced. Some preliminary in-vivo tests on healthy volunteers also are described. Current and potential future benefits of the system are discussed in Section IV.

\section{System Design and Implementation}

\section{A. Hardware and Software Description}

The system hardware consists of a computer, a DSP board based on a TMS320C6713 DSP processor (Texas Instruments Inc., Dallas, TX) and an I/O daughter board.

In addition to general-purpose DSP hardware support, all the necessary electronics for transmission (TX), reception (RX), and processing of ultrasound (US) signals are designed and implemented on the DSP board as parts of a built-in TCD unit. A field programmable gate array (FPGA) EP1S10 (Altera Inc., San Jose, CA) from the Stratix family is used to carry out hardware control and some of the digital computation tasks. A block-diagram of the data processing carried out by the TX-RX hardware, the FPGA, and the DSP processor is given in Fig. 1.

Two US probes can be connected to the two fully independent TX-RX channels of the TCD unit. For each probe, an arbitrary TX excitation burst is produced using an 8-bit digital-to-analog $(\mathrm{D} / \mathrm{A})$ converter that reads digital codes at 64 mega samples per second (MSPS) from a look-up table updated by DSP software. Currently, the transmitted time averaged acoustic power is limited at $56 \mathrm{~mW}$ when using a $2 \mathrm{MHz}$ transducer with a diameter of $14 \mathrm{~mm}$. In the receiver section, the US echoes are amplified with a

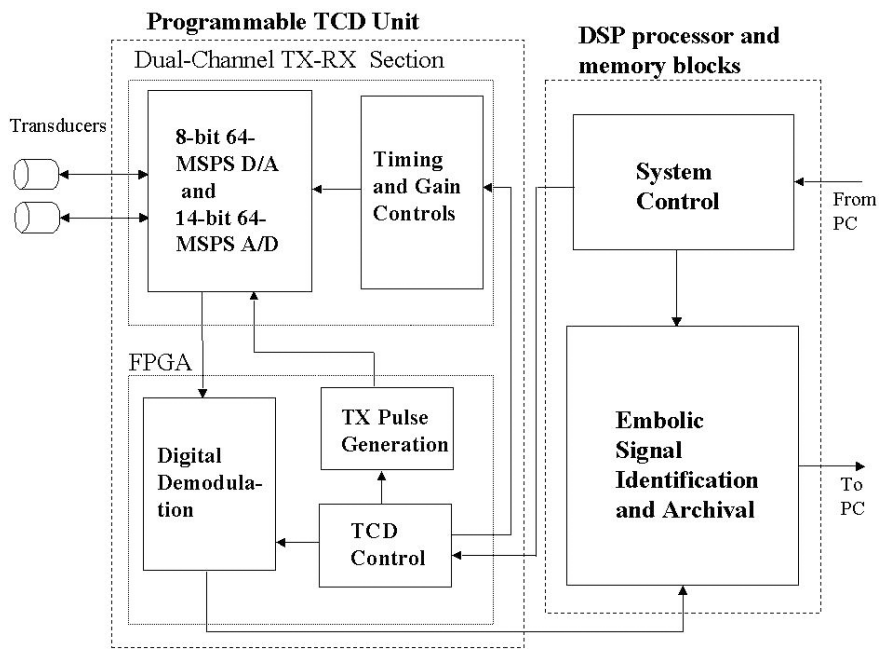

Fig. 1. Block-diagram of the ESIAS.

low noise (30 dB) amplifier followed by a programmable gain $(-10$ to $30 \mathrm{~dB})$ amplifier and analog-to-digital (A/D) converted with 14-bit resolution at 64 MSPS. This high resolution is needed to preserve the high dynamic range necessary in MES detection. The digital samples are sent to the FPGA where the digital quadrature demodulation for both channels is performed. Demodulation frequency and low-pass filter bandwidth are fully programmable.

The signals demodulated by the FPGA are converted into 32-bit floating-point format and transferred to a DSP buffer as the inputs to an automated embolus detection engine run by the TMS320C6713 processor. The DSP board is equipped with a $64 \mathrm{MB}$ SDRAM memory block in which raw data can be stored then transferred to the host computer through a USB 2.0 connection for further signal processing, storage or display purposes. This USB communication channel makes the system portable and easily in- 


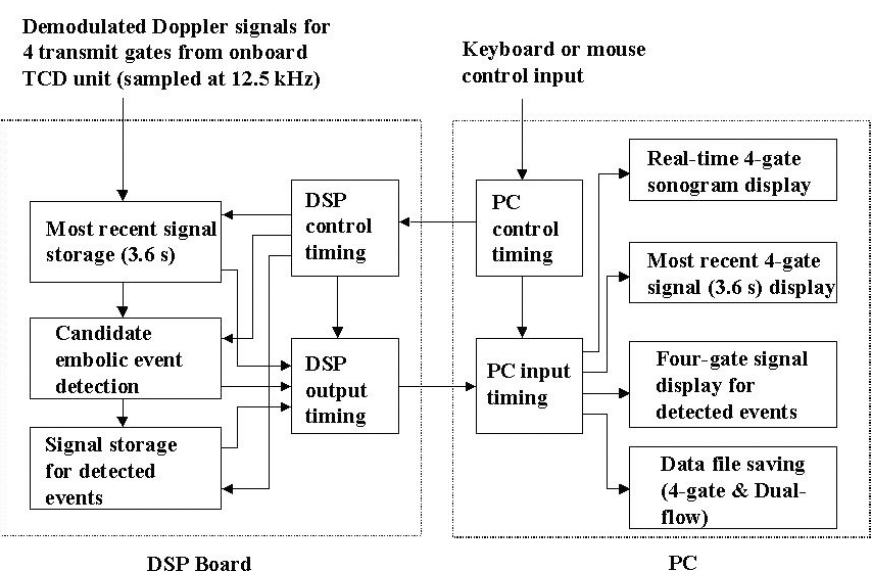

Fig. 2. Block-diagram of the system software.

stallable, and provides a peak data transfer speed higher than $50 \mathrm{MB} / \mathrm{s}$.

The audio codec PCM3003 (Texas Instruments Inc., Dallas, TX), containing two channels with 16-bit A/D and $\mathrm{D} / \mathrm{A}$ converters, is mounted onboard to meet basic audio requirements. The dual-channel output of the codec can be used to send directional Doppler signals to speakers and/or a tape recorder, and its dual-channel inputs can be used to digitize directional signals from an external Doppler signal source such as a commercial TCD machine or tape recorder. The audio facility of the system can be further exploited by attaching the I/O daughter board to the main DSP board through an onboard digital expansion port. This board is equipped with six channels of 16bit $\mathrm{A} / \mathrm{D}$ and $\mathrm{D} / \mathrm{A}$ converters, which can be used to produce analogue outputs containing multigate information (for recording purposes) or digital inputs from multiple analogue sources (for data processing purposes).

The system software is divided into two parts: the TMS320C6713 program running on the DSP board, and the MicroSoft VISUAL C ++ (MicroSoft Corp., Redmond, WA) Windows programs running on the computer. The TCD unit provides demodulated digital signals at a sampling frequency of $12.5 \mathrm{kHz}$ to the other digital processing blocks shown in Fig. 2. The tasks of the DSP programs include the control of the TCD unit, preprocessing of Doppler signals from the TCD unit, identification of embolic signals, data storage of identified events, display data preparation, and real-time data processing timing. The computer programs carry out the tasks of data transfer, real-time display, operator control interface, saving data files, and display/control timing.

\section{B. System Controls}

1. The System Input Control: A system menu allows the system input to be chosen from external sources or from the onboard TCD unit. In the former case analogue Doppler signals from external TCD machines or from tape players are captured by the on-board A/D converters, and their digital counterparts are analyzed to obtain microembolic event detection results.

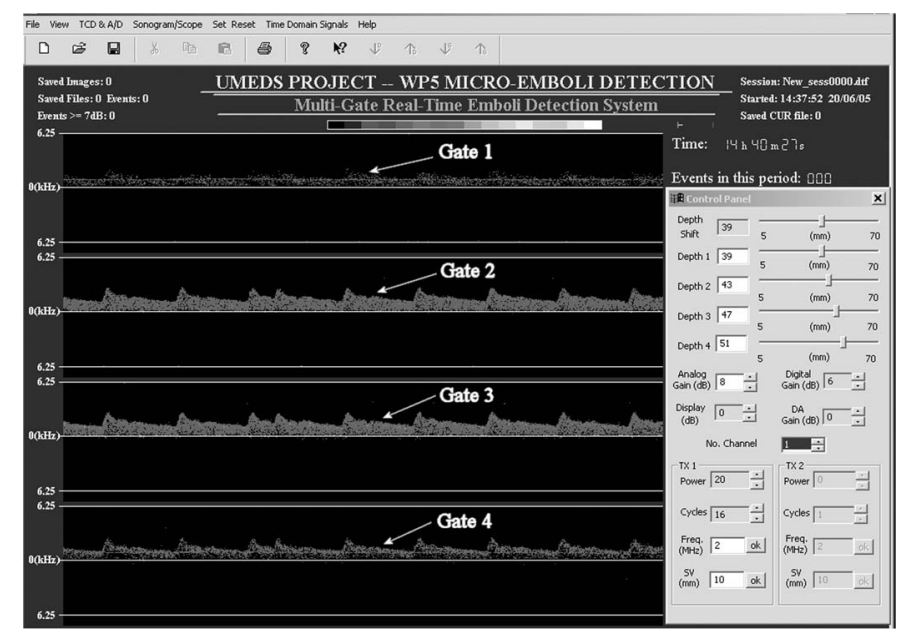

Fig. 3. Four-gate analysis of a MCA using a $2 \mathrm{MHz}$ transducer. The sonograms from gates $1,2,3$, and 4 are shown in the middle of the screen. The control panel of the system is displayed on the right-hand side, showing that four gates were set at depths of 39,43,47, and $51 \mathrm{~mm}$, respectively.

2. The TCD Controls: To make the control of the builtin TCD unit flexible and interactive, a control panel has been designed and implemented based on the Windows graphical user interface. Using the control panel, the onboard TCD unit can be programmed to run in one of two working modes: Single-Channel Mode (SCM) and DualChannel Mode (DCM). In the SCM, the TCD unit becomes a 4-gate, single probe ultrasound system. In the DCM, the TCD unit works with the two available independent TX/RX channels using two transducers. In this case two gates are available for each channel. In both modes, the TCD parameters such as transmit frequency, transmit power level, transmit cycles, sample volume, the depth of each gate, the receive analogue and digital gain controls, and the sonogram display gain control are programmable and can be changed while the system is running in realtime. The control panel is hidden by default, but it can be displayed at any time and be left open for the rest of the monitoring procedure. An example of the graphic interface is given in Fig. 3, which shows a computer image captured when the system was used in a preliminary in-vivo test.

3. System Set and Reset: The following features of the system can be set or reset during a monitoring session. A real-time clock can be set/reset to either the current computer clock or to any starting time of a tape player. The real-time counter of the detected events can be reset if the operator decides to discard any detection result during a detection period. The name of the data file can be reset if the operator wishes to save data files in several different groups.

\section{Spectral Analysis}

A digital implementation of the classic time-frequency analyzer [19] is used to produce real-time sonograms and to provide a frequency domain knowledge source for the expert system reasoning engine. After the calculation of each 
frame, the 128-sample data window (data length $10.24 \mathrm{~ms}$ ) is moved forward by 32 samples $(2.56 \mathrm{~ms})$. Thus the overlap between adjacent frames is $75 \%$, which helps to ensure no very short events are missed.

\section{Identification of Micro-Embolic Events among Artifacts and Background Signals}

The task of identification of micro-embolic events is carried out in the unit labeled 'Candidate Embolic Event Detection' (CEED) in Fig. 2, which is the key computational unit of the system. To cope with the highly complicated nature of embolic signals, the different types of artifacts, and the background Doppler signal (clutter), expert system technology was used to emulate human cognitive skills during the decision-making procedure when detecting emboli and rejecting artifacts. The unit carries out intensive signal analyses and evaluations in the time, frequency, energy, and neighborhood domains to build its knowledge source data base, which then is processed using a blackboard-type intermediate hypotheses and decision system [20]. The detailed reasoning procedure of the expert system structure is reported in [12]. The expert system was originally designed to have 14 knowledge source groups, and 76 knowledge sources derived from these groups. The blackboard evaluation used 164 rules and 45 blackboard levels in the reasoning and decision-making process, which differentiates possible embolic events from background signals and artifacts. As shown in Table II, four extra basic knowledge sources, unavailable in the previous system, have been introduced into the expert system to improve the detection performance of the system, and an additional 11 rules have been derived from these knowledge sources and added to the blackboard-type CEED unit.

\section{E. System Display}

The sonograms from four gates are displayed in realtime; each contains forward and reverse flow components. The sonogram display resolution is $5.12 \mathrm{~ms}$, and the most recent $3.58 \mathrm{~s}$ sonograms are always shown on the screen.

A real-time, updated histogram is displayed, showing detection results during each 10-minute detection period for the most recent 4 hours. During each such period, the histogram shows the total number of detected events as well as the number of those with a measured embolus-toblood-ratio (MEBR) higher than $7 \mathrm{~dB}$.

Optionally, the outputs of the onboard A/D converters or the inputs of the $\mathrm{D} / \mathrm{A}$ converters can be displayed on the screen as a digital oscilloscope. This can be useful when controlling the gain of external analogue signal sources or the magnitudes of output analogue signals.

\section{F. Signal Archival Facilities of the System}

1. Archiving Signals Corresponding to Detected Events: Each micro-embolus monitoring session is divided into multiple, 10-minute detection periods. If any event is detected during such a period, a 1 second segment of directional Doppler signal for each gate is automatically stored in the DSP data buffer. These Doppler signals correspond to the blood velocities of the forward and reverse blood flow calculated from the four programmable gates. Signal storage starts at half a second before and ends at half a second after the detection triggering point. Normally, these signals are automatically up-loaded to the computer and saved into a data file at the end of each 10-minute detection period. But, because of DSP memory limitations, if 50 events are detected before the end of the period, the computer immediately will read the raw data corresponding to these events from the DSP data buffer before saving them into a binary data file. After a data file is saved, the index part contained in the file name is updated incrementally, ready to be used for the next file. The DSP carries on the real-time task of embolic signal identification every $2.56 \mathrm{~ms}$, even when the computer is busy with saving the data from previously detected events. Up to 10,000 data files can be saved for a given file name. Dedicated software has been written to read these data files and check their contents at a later time.

The stored signal data corresponding to any event detected during a 10-minute detection period can be chosen by the operator and displayed on the computer screen as time-domain signals, which enables the operator to check the events that already have been detected.

2. Archiving the Signals Corresponding to Unknown Events Displayed on the Screen: Unknown events seen on the display screen during real-time monitoring can be further investigated and the corresponding raw data can be saved, regardless of whether these events are detected as micro-embolic candidates or not. When operators notice such an event, they can "freeze" the running realtime displays and choose any part of the displayed sonograms before displaying the corresponding eight segments (from four gates, each with forward and reverse flow components) of the time-domain signals on the screen. These time-domain signals also can be saved to a data file for further analysis at a later time. The maximum length of the signals that can be saved is 3.58 seconds $(\times 8)$, which corresponds to a full-screen display of sonograms.

\section{System Test and Preliminary Results}

\section{A. Preliminary In-Vivo Tests}

A number of preliminary in-vivo tests have been performed on healthy volunteers. These tests have been carried out to evaluate the ability of the system to work correctly in a clinical environment. The results shown in Fig. 3 were obtained from the middle cerebral artery (MCA) of a healthy volunteer using a transmit frequency of $2 \mathrm{MHz}$. Four gates, at depths of $39,43,47$, and $51 \mathrm{~mm}$, were used. Fig. 4 shows the results of monitoring both the left and 
TABLE II

The Recently Introduced Basic Knowledge Sources for the Expert System.

\begin{tabular}{|c|c|c|}
\hline Knowledge origin & $\begin{array}{l}\text { Basic knowledge sources } \\
\text { introduced }\end{array}$ & Purpose of introduction \\
\hline $\begin{array}{l}\text { The directional characteristics of } \\
\text { signals from emboli and artifacts }\end{array}$ & $\begin{array}{l}\text { The correlation coefficients cal- } \\
\text { culated using signals correspond- } \\
\text { ing to the forward and reverse } \\
\text { flows from the same gate }\end{array}$ & $\begin{array}{l}\text { To provide useful decision- } \\
\text { making information based on } \\
\text { the unidirectional property of } \\
\text { embolic signals }\end{array}$ \\
\hline $\begin{array}{l}\text { The directional characteristics of } \\
\text { signals from emboli and artifacts }\end{array}$ & $\begin{array}{l}\text { The correlation coefficients cal- } \\
\text { culated using the MEBRs corre- } \\
\text { sponding to the forward and re- } \\
\text { verse flows from the same gate }\end{array}$ & $\begin{array}{l}\text { To provide useful decision- } \\
\text { making information based on } \\
\text { the unidirectional property of } \\
\text { embolic signals }\end{array}$ \\
\hline $\begin{array}{l}\text { The spatial distribution of the } \\
\text { receive gates }\end{array}$ & $\begin{array}{l}\text { The correlation coefficients cal- } \\
\text { culated using signals correspond- } \\
\text { ing to forward or reverse flow } \\
\text { pairs from different gates }\end{array}$ & $\begin{array}{l}\text { To provide useful decision- } \\
\text { making information based on } \\
\text { the non-traveling property of } \\
\text { artifact signals }\end{array}$ \\
\hline $\begin{array}{l}\text { The spatial distribution of the } \\
\text { receive gates }\end{array}$ & $\begin{array}{l}\text { The correlation coefficients cal- } \\
\text { culated using the MEBRs corre- } \\
\text { sponding to the forward or re- } \\
\text { verse flow pairs from different } \\
\text { gates }\end{array}$ & $\begin{array}{l}\text { To provide useful decision- } \\
\text { making information based on } \\
\text { the nontraveling property of } \\
\text { artifact signals }\end{array}$ \\
\hline
\end{tabular}

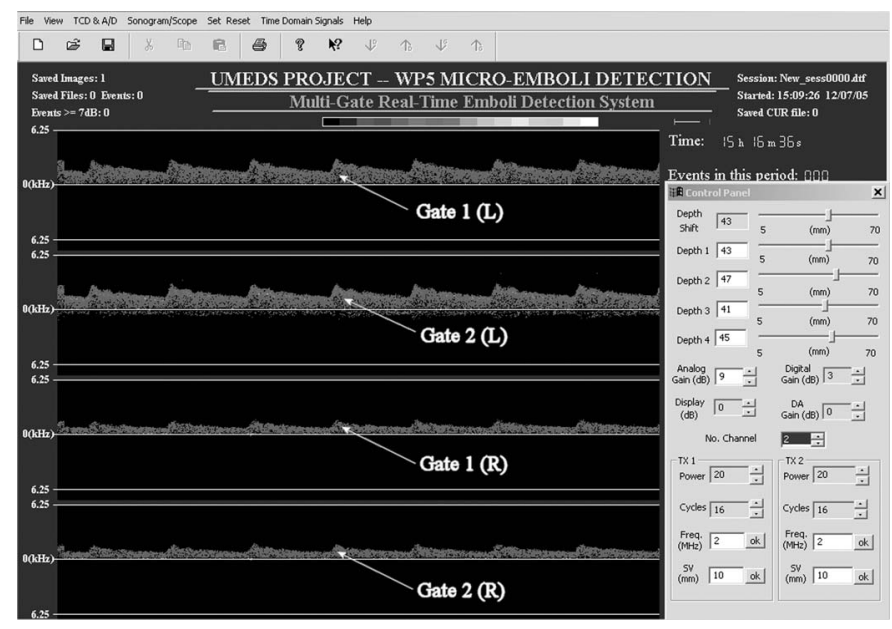

Fig. 4. Bilateral monitoring of MCAs. Two 2-MHz transducers were used to obtain sonograms from a left MCA [Gate 1 (L) and Gate 2 $(\mathrm{L})$ ] and a right MCA [Gate $1(\mathrm{R})$ and Gate $2(\mathrm{R})]$. Gate depths for the left MCA were 43 and $47 \mathrm{~mm}$, and for the right MCA, 41 and $45 \mathrm{~mm}$.

right MCAs simultaneously. Signals were obtained from the left and right temporal windows of a 28-year old volunteer using two $2 \mathrm{MHz}$ probes, with two gates for each probe.

Fig. 5 shows a typical result obtained during simultaneous monitoring of $\mathrm{MCA}$ and common carotid arteries (CCA). Two probes, one of $2 \mathrm{MHz}$ and another of $8 \mathrm{MHz}$, were used here for simultaneous intracranial and extracranial investigation.

\section{B. Experimental In-Vitro Test}

Preliminary in-vitro experiments have been carried out to test the sensitivity performance of the embolus detec-

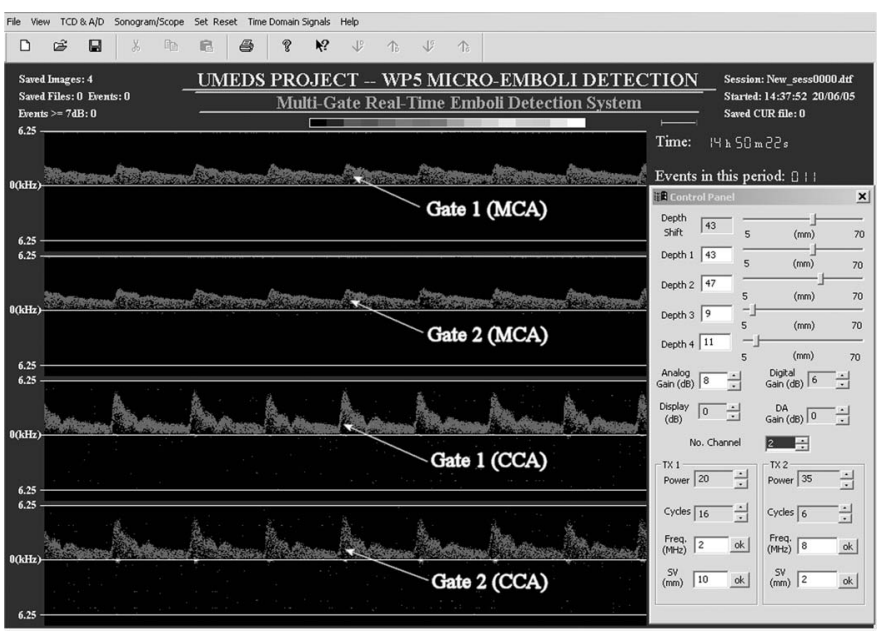

Fig. 5. Simultaneous monitoring of a MCA and a CCA. A $2 \mathrm{MHz}$ transducer was used to obtain the two-gate sonograms from the $\mathrm{MCA}$, and an $8 \mathrm{MHz}$ transducer was used for the two-gate sonograms from the CCA. The gate depths are 43 and $47 \mathrm{~mm}$ for the MCA, 9 and $11 \mathrm{~mm}$ for the CCA.

tion system (single gate only) using an embolus flow phantom. The phantom consisted of a gear pump and a tank $\left(\mathrm{L} \times \mathrm{H} \times \mathrm{W}=25 \times 19.5 \times 10 \mathrm{~cm}^{3}\right)$ filled with tissue mimicking material (TMM). The pump generated a steady flow of a blood mimicking fluid (BMF) through a 3-mm wall-less channel within the TMM, at a velocity of approximately $35 \mathrm{~cm} \mathrm{~s}^{-1}$. Details of the TMM, BMF, and construction of the wall-less TMM channel can be found in [21]. Artificial emboli consisting of small particles of TMM were injected into the flow to produce embolus-like Doppler signals. In addition to these particles, air bubbles also were introduced to simulate gaseous emboli. As the main purpose was to test sensitivity at this stage, no artifacts were created during the experiment. A $2 \mathrm{MHz}$ transducer was 


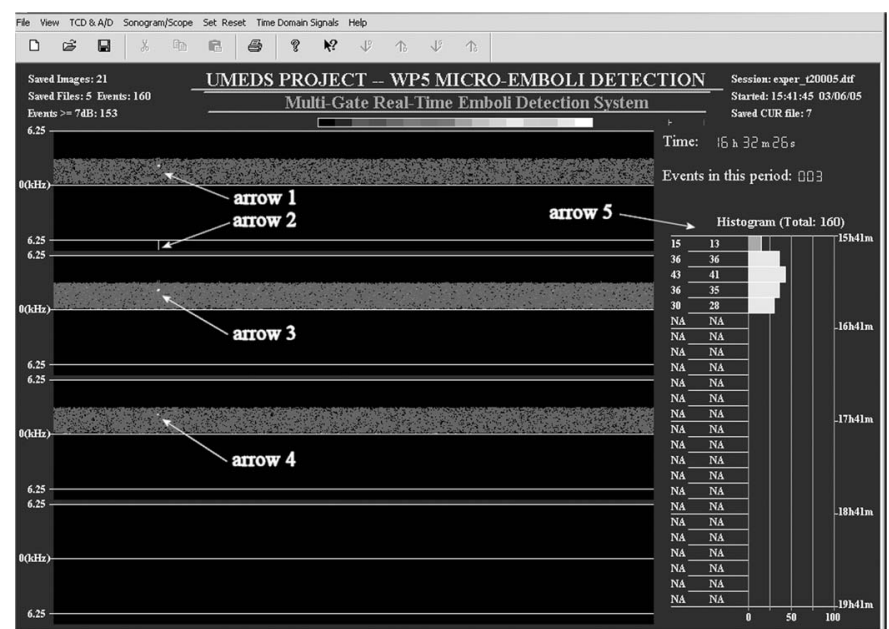

Fig. 6. Example of a detected event when the system was tested using a flow phantom. The top most sonogram corresponds to gate 1, which shows that a signal from an embolus-like particle (arrow 1) was detected by the system, and an indicator line automatically displayed (arrow 2). The embolic signal can be seen on the sonograms corresponding to gates 2 and 3 (arrows 3 and 4), but not on the sonogram from gate 4 , which was deliberately set outside the vessel of the flow phantom. The histogram of detected events (arrow 5) shows that the system detected a total of 160 candidate embolic events in the first 50 minutes. The two columns of figures to the left of the histogram bars detail the total number of embolic events detected in any one 10-minute period (left), and the number of events with an MEBR greater than $7 \mathrm{~dB}$ (right). The depths of the four receive gates were $46,49,52$, and $15 \mathrm{~mm}$, respectively.

connected to the on-board TCD unit; it was fixed on top of the tank and angled nearly parallel to the flow direction. A 10-mm sample volume was used, and the depth was set at $45 \mathrm{~mm}$. During the automated detection, the demodulated directional analogue Doppler signals produced by the on-board TCD unit were recorded in real time using a tape recorder that had its clock synchronized to that of the monitoring system. The recorded $420 \mathrm{~s}$ signal was later manually examined by an experienced human expert, and all events, irrespective of their MEBR, used in a comparison with those detected by the automated system.

The results of the laboratory tests indicate that, without using an MEBR threshold, the system reached a sensitivity of $93.60 \%$ and a specificity of $99.99 \%$ (recorded signal duration, $420 \mathrm{~s}$; all candidate events, 10500; true embolic events, 203; mean MEBR of the true embolic events, $14.9 \mathrm{~dB}$ ) when compared to a human observer. An example of the detection results saved as a screen image is given in Fig. 6, showing signals from an embolus-like particle detected by the system (this image was saved during a separate laboratory test).

Fig. 7 shows an example of an embolus traveling through gates $1-3$. The grey rectangle defines the time period for which the time-domain signals from the four gates were displayed. Signals from an embolus-like particle can be seen in the first three gates, with evident time delays among them. No signal appears on gate 4 , which was deliberately set outside the vessel in the phantom. Figs. 8 and 9 were obtained after an artifact was created by touching the

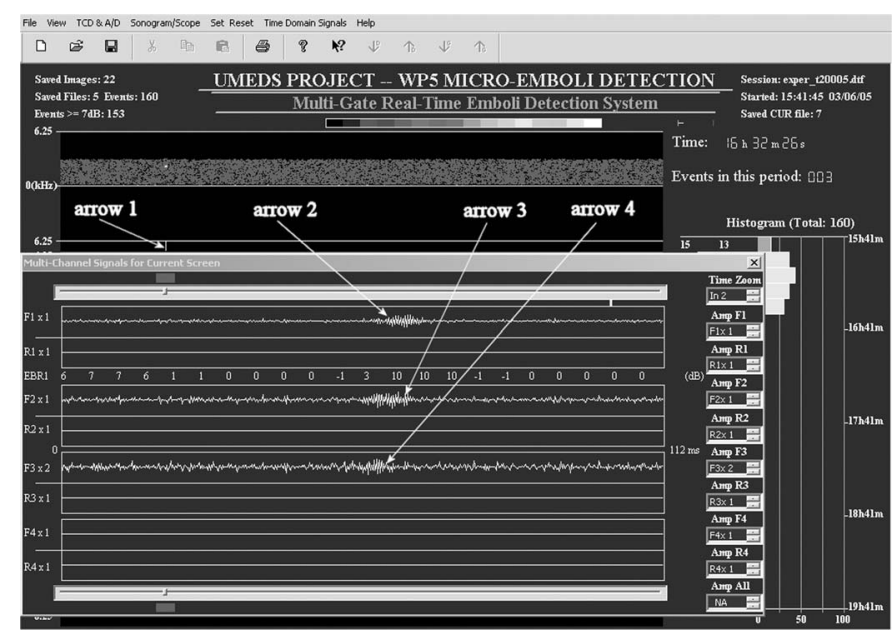

Fig. 7. Example of embolic event traveling among three gates. This captured computer image is the same as the one shown in Fig. 6, except that time signals were displayed to show their mutual delay. A time interval was selected from the sonogram display as indicated by a rectangle (below arrow 1) to display time-domain signals from this period. The time-domain signals from the detected embolic event can be seen at gates $1-3$ (arrows $2-4$ ).

probe. The artifact appears in the sonograms for all gates, including gate 4 that was, also in this case, set outside the vessel.

\section{Initial Tests on the Improvement of Embolus Detection}

In order to explore the potential for the improvement in performance using the new knowledge sources, preliminary tests were carried out to analyze data files containing multigate and bidirectional Doppler signals. These data files were recorded digitally from a volunteer and from a patient during cardiac surgery, using the new system (but with a version of software written primarily for recording purposes).

The data from the healthy volunteer were embolusfree, but they contain a number of deliberately introduced artifacts such as talking, probe taping/moving, etc. The depths of gates 1 and 2 were set at 44 and $25 \mathrm{~mm}$ during the $162 \mathrm{~s}$ recording. The data from the patient were recorded during surgery to replace an aortic valve. The recorded signal duration was $406 \mathrm{~s}$, with the depths of gates 1 and 2 being set at 45 and $30 \mathrm{~mm}$, respectively. A $2 \mathrm{MHz}$ transducer and a sampling frequency of $8.06 \mathrm{kHz}$ were used during both recordings.

The Doppler signals generated from the data files were analyzed in real time using both the original expert system (i.e., the one reported in [12]) and an improved version using the basic knowledge sources shown in Table II (this is currently in the form of separate stand-alone embolus detection software, to be finalized and incorporated into the TCD integrated system). The results from the original and novel systems were carefully examined by an experienced human expert, and the sensitivity/specificity performances were compared. 


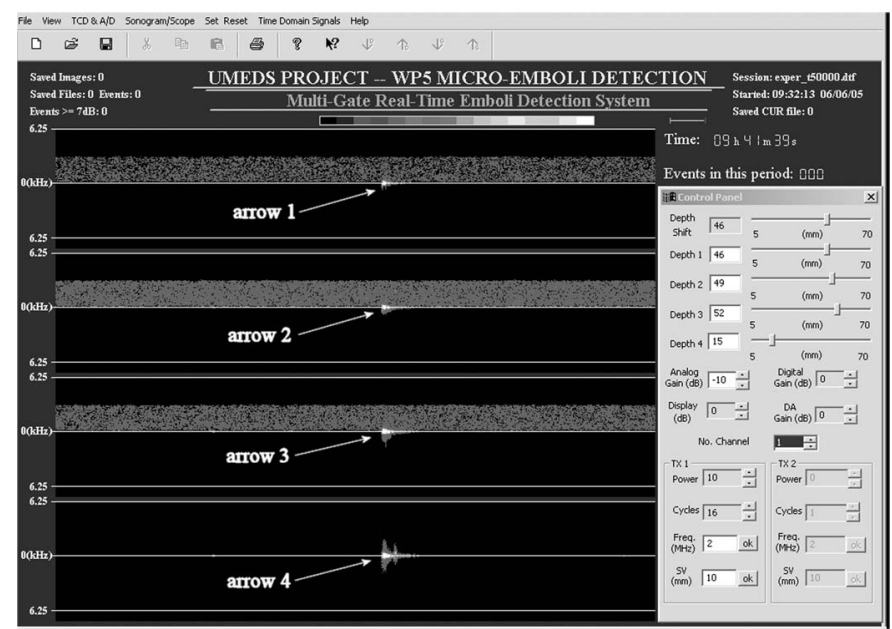

Fig. 8. An example of signals due to an artifact. The four gates were arranged in the same configuration as in Fig. 6. An artifact was created by touching the probe that was fixed at the top of the flow phantom. It can be seen that the artifact event appeared in the sonograms for all gates (arrows 1-4), including gate 4 (arrow 4), which was deliberately set outside the vessel of the flow phantom. The figure also shows that the artifact produced frequency components that were symmetric about the base line.

The results showed that, using a $7 \mathrm{~dB}$ MEBR threshold, the new system reached a sensitivity of $98.68 \%$ and a specificity of $100.00 \%$ (all candidate events, 7877 ; true embolic events, 302; mean MEBR of the true embolic events, $28.2 \mathrm{~dB}$ ) compared with the corresponding performance of the original system of $89.07 \%$ and $99.99 \%$, respectively.

An example of the detection results from the novel system is given as a saved screen image in Fig. 10, showing a group of embolic signals detected by the new expert system.

\section{Discussion}

The TCD integrated ESIAS was designed and implemented with state-of-art DSP hardware, into which spectral analysis and expert system reasoning algorithms have been integrated. The system has been adapted from a previously described, single-gate embolic event detection system to provide further improvement in system performance by gathering bidirectional information from multiple gates.

The TCD integrated ESIAS has the following advantages over the system reported in [17]:

- Flexibility. The CEED of the system can be configured to directly process digital data from the on-board TCD unit. It also can be configured to process data from an external TCD unit, using the on-board A/D converters. The on-board FPGA can be programmed to take over some of the workload from the DSP processor if necessary.

- User-friendly system control. The on-board TCD and the embolus detection and archiving functions can be

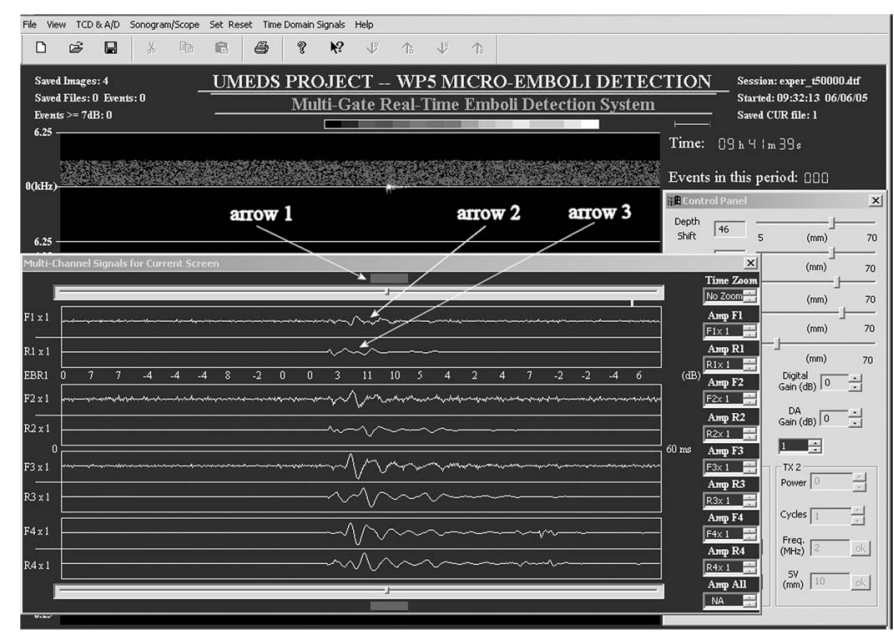

Fig. 9. An example of signals from an artifact. This captured computer image is the same as the one shown in Fig. 8, except that time-domain signals are displayed. The image shows that a part of the sonograms was selected (indicated by the grey rectangle-arrow 1 ), and the corresponding time-domain signals for the four gates were displayed. Signals from an artifact can be seen in both flow directions in the first gate (arrows 2 and 3). Similarly, signals from the artifact appeared in all other gates with no time delay among them (displayed below those of the first gate), including gate 4, which was deliberately set outside the vessel of the flow phantom.

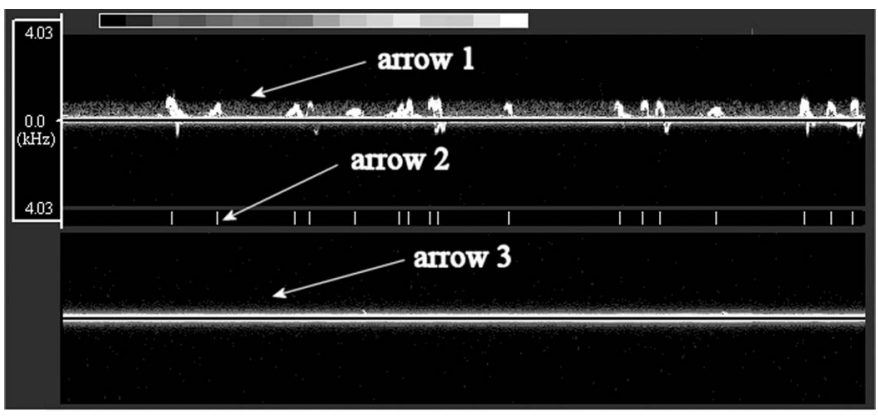

Fig. 10. Example of a group of events detected using multigate and dual-directional information. The top sonogram corresponds to gate 1 (arrow 1), which shows that a group of embolic signals were detected by the system, and the corresponding indicator lines automatically displayed (arrow 2). The embolic signals do not appear on the bottom sonogram corresponding to gate 2 (arrow 3), which was deliberately set outside the vessel of the patient. These results were obtained by playing back and processing data files, which were digitally recorded during surgery for an aortic valve substitution. The depths of gates 1 and 2 were set at 45 and $30 \mathrm{~mm}$, respectively.

controlled more easily and intuitively, using a single display-and-control center.

- Achievement of floating-point dynamic range integrity of the entire system. The fixed-point data I/O interface (usually a $\mathrm{D} / \mathrm{A}$ followed by an $\mathrm{A} / \mathrm{D}$ structure) between an external TCD unit and the embolus detection unit is eliminated when using the on-board TCD. - Small dimensions. A programmable TCD unit and a real-time MES identification and archiving system have been integrated into one state-of-art device $\left(23 \times 21 \times 6.2 \mathrm{~cm}^{3}\right)$. 
Although our previously described, single-gate system reached very high levels of specificity in in-vivo tests (99.2\%), during long monitoring sessions there may be very large numbers of significant increases in Doppler signal power caused by mechanisms unrelated to emboli, and therefore, even higher levels of specificity are desirable. The current system allows us to develop additional strategies for rejecting artifacts based both on the time relationship between the signals in separate gates and their directional properties. Comparison of Figs. 7 and 9 shows how, in the case of simple events, there are clear differences between the characteristics of embolic signals and artifacts. Embolic signals show a progressive time delay between their arrivals in adjacent gates and are unidirectional, and artifacts appear simultaneously in all gates (including the gate placed outside the vessel) and are bidirectional [8], [11], [14], [22]-[27]. The inclusion of these properties as inputs into our expert reasoning system will further enhance its high performance levels.

\section{CONCLUSIONS}

A real-time TCD integrated embolic signal identification/archival system has been designed and implemented. The system is based on state-of-art hardware components including a TMS320C6713 DSP processor, and a novel expert system software structure. The integrated, programmable TCD unit can be configured either as a singlechannel, four-gate ultrasound system or as a dual-channel, dual-gate system using two ultrasound transmit frequencies. The system has provided real-time sonogram display and real-time automated embolic signal acquisition for bidirectional signal pairs from four gates.

In-vitro experiments have been carried out to test the sensitivity performance of the system (single gate only) using an embolus flow phantom. The preliminary experimental results show a sensitivity of $93.6 \%$ and a specificity of $99.99 \%$ when using the system to identify the solid and gaseous emboli introduced into the flow system of the phantom.

Initial in-vivo experiments indicate that, by analyzing multigate and bidirectional signals, embolus detection performance can be further improved. For the short-signal sample tested, sensitivity and specificity improved from $89.07 \%$ and $99.99 \%$, to $98.68 \%$ and $100.00 \%$, respectively. The new embolus detection program will require some further improvement before being evaluated using a much larger data set.

\section{ACKNOWLEDGMENTS}

The authors thank Dr. K. Ramnarine and Dr. J. Gittings for their help with the in-vitro experiments and P. Fidanzati for assistance in software development.

\section{REFERENCES}

[1] M. P. Spencer, "Detection of cerebral arterial emboli," in Transcranial Doppler Ultrasonography. D. W. Newell and R. Aaslid, Eds. New York: Raven, 1992, pp. 215-230.

[2] D. H. Evans, "Detection of microemboli," in Transcranial Doppler Ultrasonography. 2nd ed. V. L. Babikian and L. R. Wechsler, Eds. Boston: Butterworth Heinemann, 1999, pp. 141155.

[3] H. Markus, "Microembolic signal detection in cerebrovascular disease," in Transcranial Doppler Ultrasonography. 2nd ed. V. L. Babikian and L. R. Wechsler, Eds. Boston: Butterworth Heinemann, 1999, pp. 167-174.

[4] R. Dittrich, M. A. Ritter, and D. W. Droste, "Microembolus detection by transcranial Doppler ultrasonography," Eur. J. Ultrasound, vol. 16, pp. 21-30, Nov. 2002.

[5] D. H. Evans, "Ultrasonic detection of cerebral emboli," in Proc. IEEE Ultrason. Symp., 2003, pp. 316-326.

[6] H. Markus, A. Loh, and M. M. Brown, "Computerized detection of cerebral emboli and discrimination from artifact using Doppler ultrasound," Stroke, vol. 24, pp. 1667-1672, Nov. 1993.

[7] M. Siebler, G. Rose, M. Sitzer, A. Bender, and H. Steinmetz, "Real-time identification of cerebral microemboli with US feature detection by a neural network," Radiology, vol. 192, pp. 739-742, Sep. 1994.

[8] D. Georgiadis, A. Wenzel, H. R. Zerkowski, S. Zierz, and A. Lindner, "Automated intraoperative detection of Doppler microembolic signals using the bigate approach," Stroke, vol. 29, pp. 137-139, Jan. 1998.

[9] E. Roy, S. Montresor, P. Abraham, and J. L. Saumet, "Spectrogram analysis of arterial Doppler signals for off-line automated hits detection," Ultrasound Med. Biol., vol. 25, pp. 349-359, Mar. 1999.

[10] V. Kemeny, D. W. Droste, S. Hermes, D. G. Nabavi, G. SchulteAltedorneburg, M. Siebler, and E. B. Ringelstein, "Automatic embolus detection by a neural network," Stroke, vol. 30, pp. 807-810, Apr. 1999.

[11] W. H. Mess, B. M. Titulaer, and R. G. Ackerstaff, "A new algorithm for off-line automated emboli detection based on the pseudo-wigner power distribution and the dual gate TCD technique," Ultrasound Med. Biol., vol. 26, pp. 413-418, Mar. 2000.

[12] L. Fan, D. H. Evans, and A. R. Naylor, "Automated embolus identification using a rule-based expert system," Ultrasound Med. Biol., vol. 27, pp. 1065-1077, Aug. 2001.

[13] D. Georgiadis, F. Uhlmann, M. Astler, S. Cencetti, and S. Zierz, "Automated identification of Doppler microembolic signals: Comparison of two techniques," Neurol. Res., vol. 22, pp. 738-740, Oct. 2000.

[14] G. Devuyst, G. A. Darbellay, J. M. Vesin, V. Kemény, M. Ritter, D. W. Droste, C. Molina, J. Serena, R. Sztajzel, P. Ruchat, C. Lucchesi, G. Dietler, E. B. Ringelstein, P. A. Despland, and J. Bogousslavsky, "Automatic classification of HITS into artifacts or solid or gaseous emboli by a wavelet representation combined with dual-gate TCD," Stroke, vol. 32, pp. 2803-2809, Dec. 2001.

[15] R. Brucher and D. Russell, "Automatic online embolus detection and artifact rejection with the first multifrequency transcranial Doppler," Stroke, vol. 33, pp. 1969-1974, Aug. 2002.

[16] M. Cullinane, Z. Kaposzta, S. Reihill, and H. S. Markus, "Online automated detection of cerebral embolic signals from a variety of embolic sources," Ultrasound Med. Biol., vol. 28, pp. 1271-1277, Oct. 2002.

[17] L. Fan, D. H. Evans, A. R. Naylor, and P. Tortoli, "Real-time identification and archival of microembolic Doppler signals using a knowledge-based system," Med. Biol. Eng. Comput., vol. 42, pp. 193-200, Mar. 2004.

[18] S. Marvasti, D. Gillies, F. Marvasti, and H. S. Markus, "Online automated detection of cerebral embolic signals using a waveletbased system," Ultrasound Med. Biol., vol. 30, pp. 647-653, May 2004.

[19] L. Cohen, "Time-frequency distributions-A review," Proc. IEEE, vol. 77, pp. 941-981, July 1989.

[20] P. Jackson, Introduction to Expert Systems. 2nd ed. Harlow, UK: Addison-Wesley, 1990, pp. 368-383.

[21] K. V. Ramnarine, T. Anderson, and P. R. Hoskins, "Construction and geometric stability of physiological flow rate wall-less stenosis phantoms," Ultrasound Med. Biol., vol. 27, no. 2, pp. 245-250, 2001. 
[22] J. L. Smith, D. H. Evans, L. Fan, P. R. F. Bell, and A. R. Naylor, "Differentiation between emboli and artefacts using dual-gated transcranial Doppler ultrasound," Ultrasound Med. Biol., vol. 22, pp. 1031-1036, 1996.

[23] D. Georgiadis, J. Goeke, M. Hill, M. König, D. G. Nabavi, F. Stögbauer, P. Zunker, and E. B. Ringelstein, "A novel technique for identification of Doppler microembolic signals based on the coincidence method: In vitro and in vivo evaluation," Stroke, vol. 27, pp. 683-686, Apr. 1996.

[24] G. D. Nabavi, D. Georgiadis, T. Mumme, P. Zunker, and E. B. Ringelstein, "Detection of microembolic signals in patients with middle cerebral artery stenosis by means of a bigate probe. A pilot study," Stroke, vol. 27, pp. 1347-1349, Aug. 1996.

[25] J. Molloy and H. S. Markus, "Multigated Doppler ultrasound in the detection of emboli in a flow model and embolic signals in patients," Stroke, vol. 27, pp. 1548-1552, Sep. 1996.

[26] R. Brucher, D. Russell, and H. Frenkenberger, "Automatic cerebral embolus detection using dual-gate Doppler," in New Trends in Cerebral Hemodynamics and Neurosonology. J. Klingelhofer, E. Bartels, and E. B. Ringelstein, Eds. Amsterdam: Elsevier Science, 1997, pp. 215-230.

[27] D. Georgiadis, F. Uhlmann, A. Lindner, and S. Zierz, "Differentiation between true microembolic signals and artefacts using an arbitrary sample volume," Ultrasound Med. Biol., vol. 26, pp. 493-496, Mar. 2000.

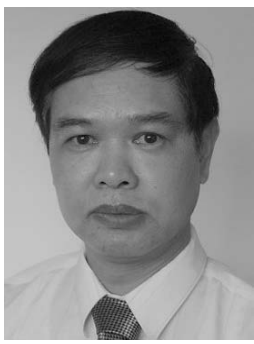

Lingke Fan (M'99) received his B.Eng. and M.Eng. degrees in acoustic engineering from Harbin Engineering University (HEU), Peoples Republic of China, in 1982 and 1985, respectively. He received his $\mathrm{Ph} . \mathrm{D}$. degree in ultrasound signal processing in 1996 from the University of Leicester, Leicester, England.

He worked as a teaching assistant then a lecturer in the HEU from 1985 to 1989 . He was a visiting researcher in University of Technology, Loughborough, England, from 1989 to 1991. He worked in University Hospitals of Leicester, National Health Service Trust (UHL NHS Trust), Leicester, England, from 1991 to 1996, as a research scientist. He was a lecturer in Singapore Polytechnic, Singapore, from 1996 to 1998. He rejoined the Department of Medical Physics of the UHL NHS Trust in 1998, where he currently works as a senior physicist. Dr. Fan is a chartered engineer registered with the Engineering Council (UK).

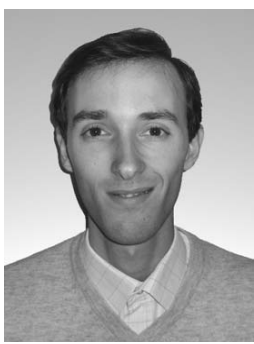

Enrico Boni graduated in electronic engineering in 2001 at the University of Florence, Italy. He received his Ph.D. degree from the same University in 2005. He currently holds a post-Doctoral position at the Microelectronic Systems Design Laboratory of the University of Florence.

His interests include analog/digital systems design and the development of digital signal processing algorithms for application to Doppler ultrasound with emphasis on microemboli detection.

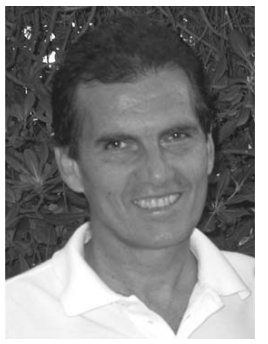

Piero Tortoli (M'91-SM'96) received the Laurea degree in Electronic Engineering from the University of Florence in 1978. Since then, he has been with the Electronics and Telecommunications Department of the University of Florence, where he is currently a full professor of Electronics. His main interests are in the area of signal processing systems with application to biomedical instrumentation. He has published over 120 papers related to his research activity on ultrasonic imaging and Doppler techniques.

Piero Tortoli is on the Editorial Board of Ultrasound in Medicine and Biology. He has been a member of the Technical Program Committee of international conferences, including the IEEE International Ultrasonics Symposium. He was also chairman of the 22nd International Symposium on Acoustical Imaging (1995), co-Chairman of the ICB Seminar on Ultrasound in Biomeasurements, Diagnostics and Therapy (1998), and Chairman of the 12th New England Doppler Conference (2003).

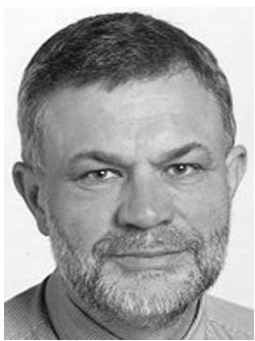

David H. Evans (SM'92) was born in Cheshire, England, in 1950. He received a B.Sc. degree in physics from the University of Surrey, Guildford, England, in 1972, a Ph.D. degree in medical physics from the University of Leicester, Leicester, England, in 1979, and a D.Sc. degree from the University of Surrey in 2001 for his contribution to Doppler Ultrasound in Medicine.

He has worked in Leicester, England, since 1974, except for a 2-year period between 1980 and 1982 when he was a visiting professor of biomedical engineering at the Federal University of Rio de Janeiro, Brazil. He was appointed to the Chair of Medical Physics at the University of Leicester in 1990, and he is an Honorary Consultant in the University Hospitals of Leicester, National Health Service Trust, where he heads the Department of Medical Physics.

Dr. Evans is a Chartered Physicist, a Chartered Scientist, a Fellow of the Institute of Physics, a Fellow of the Institute of Physics and Engineering in Medicine, a Senior Member of IEEE, and an Honorary member of the British Medical Ultrasound Society, having served as Honorary Secretary, as Honorary Treasurer, and as President from 1996-1998. He is currently President of the European Federation of Societies for Ultrasound in Medicine and Biology, previously having served as its Honorary Secretary. He was Editor of the journal Physiological Measurement from 1993 to 1997, and Instrumentation and Techniques Editor of the Journal of Clinical Ultrasound from 1993 to 1996 . 